\title{
Research of Particle Transport with External Driving Force and Entropy Barrier
}

\author{
S.X. LI ${ }^{a, b}$, Z.P. ZHANG ${ }^{b, *}$ AND D.L. CHEN ${ }^{a}$ \\ ${ }^{a}$ School of Physics and Electronic Science, Guizhou Education University, Guiyang 550018, China \\ ${ }^{b}$ College of Big Data and Information Engineering, Guizhou University, Guiyang 550025, China \\ (Received January 21, 2016; revised version March 27, 2016; in final form April 6, 2016) \\ Transport of Brownian particle moving along a three-dimensional throat-like channel is investigated in the \\ presence of an external constant force. The solution of the Fick-Jacobs equation in the situation is solved, and \\ the probability current density and particle current describing the motion of particle are obtained. It is found that \\ entropy barrier and external force can reverse the direction of particle current. The motion of Brownian particle \\ can be tuned by the entropy barrier and the external force.
}

DOI: 10.12693/APhysPolA.129.1105

PACS/topics: 05.40.Jc, 05.40.-a, 05.10.Gg, 05.60.Cd

\section{Introduction}

Transport of Brownian particles within channels, microfluidic devices, or other biological systems is a fundamental problem, which depends on the size of particle, environment, various noise, interaction between particles, and the structure of the system etc. [1-10]. Effective control of particle is important and has gained increasing attentions, and the results have implications in processes such as particle separation, molecular motors, Brownian pumps, and electrophoretic transport [10-12].

Earlier research by Zwanzig [13] was focused upon transport in a tube or channel of varying cross- section, in the absence of external force fields, with socalled Fick-Jacobs equation. Entropy barrier concept is introduced to describe the diffusion of particle. Entropy barrier produced by varying channel can modify transport properties of particle. This treatment is useful that the motion of particle in two-dimensional (2D) or three-dimensional (3D) channel can be reduced to an effective one-dimensional (1D) transport model [13, 14]. The Fick-Jacobs equation is generalized by Reguera and Rubi [14], which can describe the system including external potential and entropy barrier. Similar researches of transport in the presence of external potential and periodic entropy potential have attracted a lot of attention in recent years [3-10]. In their researches, transport properties can be analyzed by means of Fokker-Planck or Fick-Jacobs equations. However, the solutions of these equations are in general difficult and may sometimes constitute a formidable task.

The recent researches focus upon the motion of Brownian particle in a periodic channel with complex external driving force $[2-10]$. These researches are achieved by using a quasi-steady state limit to solve the Fick-Jacobs

\footnotetext{
*corresponding author; e-mail: zpzhang@gzu.edu.cn
}

equation, and have obtained the effective mobility and effective diffusion coefficient. In this letter, we investigate the motion of Brownian particle in a 3D channel with an external constant force. The solution of the Fick-Jacobs equation in the situation is solved, and the splitting effect based on the entropy barrier and the external force is analyzed.

\section{Theoretical analysis}

We consider Brownian particle moving in a throat-like channel (Fig. 1). The over-damped dynamics of Brownian particle in a 3D channel, driven by a constant force $F$, directed along the axis of the channel, can be described by the Langevin equation [1]:

$$
\eta \frac{\mathrm{d} \boldsymbol{r}}{\mathrm{d} t}=\boldsymbol{F}+\sqrt{\eta k T} \boldsymbol{\xi}(t)
$$

where $\boldsymbol{r}$ is the position vector of particle, $\eta$ is the friction coefficient of particle, $k$ is the Boltzmann constant, $T$ is temperature, $F=|\boldsymbol{F}|$ is a constant force in $x$ direction, and $\boldsymbol{\xi}(t)$ is a Gaussian white noise with zero mean and correlation function $\left\langle\xi_{i}(t) \xi_{j}\left(t^{\prime}\right)\right\rangle=2 \delta_{i j} \delta\left(t-t^{\prime}\right)$ for $i$, $j=x, y, z$. The reflecting boundary condition is imposed at the channel wall $[1,3]$.

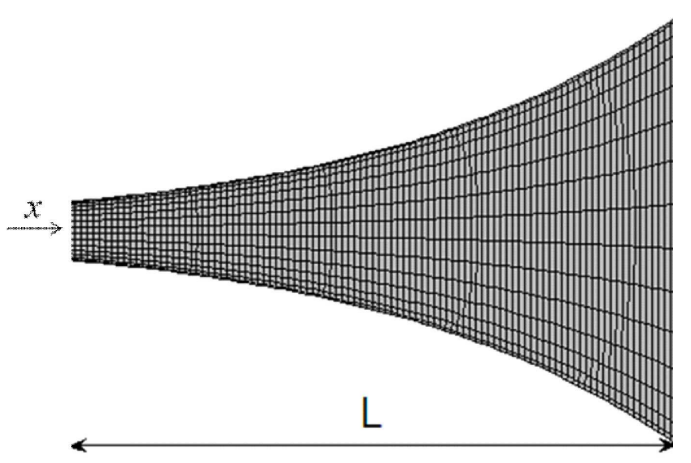

Fig. 1. Diagram of the three-dimensional throat-like channel, the radius of the channel $\omega(x)$ is defined with Eq. (2), $L$ is length of channel. 
The shape of the channel is described by

$$
\omega(x)=\sqrt{\exp (a x+b) / \pi} \quad(-L / 2 \leq x \leq L / 2),
$$

where $\omega(x)$ is radius of the channel $a$ and $b$ are geometric characteristic parameters of the channel physical unit of $a$ is $\mu \mathrm{m}^{-1}$ and the size of $a$ controls the slope of the channel wall, $L$ is the length of the channel, the size of $L$ can be selected according to actual situation.

The movement of Brownian particle moving along the axis of the 3D channel can be described by the FickJacobs equation, by following the researches $[1,2,14]$, the description of this system can be given

$$
\begin{aligned}
& \frac{\partial P(x, t)}{\partial t}=\frac{\partial}{\partial x}\left[D(x) \frac{\partial P(x, t)}{\partial x}\right. \\
& \left.+\frac{D(x)}{k T} \frac{\partial V(x, t)}{\partial x} P(x, t)\right]=-\frac{\partial J(x, t)}{\partial x},
\end{aligned}
$$

where $V=E-T S=-F x-T k \ln h(x)$ is the free energy including the energy $E=-F x$ and the entropy $S=k \ln h(x), h(x)$ is the dimensionless transverse crosssection $\pi[\omega(x) / L]^{2}$ of the three dimensions channel entropy $S$ determined by the channel of varying crosssection is often described as entropy barrier [14, 15]. $D(x)=D_{0} /\left(1+\omega^{\prime}(x)^{2}\right)^{1 / 2}$ is position-dependent diffusion coefficient $[1,3]$, where $D_{0}=k T / \eta . J(x, t)$ is the probability current density, $P(x, t)$ is the probability density for the particle at position $x$ and at time $t$.

If the characteristic parameters $a \leq 1$ and $b \leq 1$, the diffusion coefficient approximate to constant $D(x) \approx D_{0}$, in this approximation, combining Eq. (2) and Eq. (3) can be given as

$$
\frac{\partial P(x, t)}{\partial t}=\frac{\partial}{\partial x}\left[D_{0} \frac{\partial P(x, t)}{\partial x}-D_{0}\left(\frac{F}{k T}+a\right) P(x, t)\right] .
$$

Note that the equation does not depend on characteristic parameter $b$. When the initial condition is $P(x, 0)=$ $\delta(x)$, the solution of this equation is given by

$$
P(x, t)=\sqrt{\frac{1}{4 \pi D_{0} t}} \exp \left(\frac{-\left[x-D_{0}\left(\frac{F}{k T}+a\right) t\right]^{2}}{4 D_{0} t}\right) .
$$

According to Eq. (5) we can obtain the ratio

$$
p(x)=\frac{P(x, t)}{P(-x, t)}=\exp \left(\left(\frac{F}{k T}+a\right) x\right), 0 \leq x \leq L / 2 .
$$

Equation (6) indicates that this ratio does not depend on time and only involves the free energy.

In the absence of an external force the free energy is pure entropy and Eq. (6) becomes

$$
p(x)=\exp (a x), 0 \leq x \leq L / 2 .
$$

According to Eq. (3) and Eq. (5) we can obtain the probability current density

$$
\begin{aligned}
& J(x, t)=\sqrt{\frac{1}{16 \pi D_{0} t}} \exp \left(\frac{-\left[x-D_{0}\left(\frac{F}{k T}+a\right) t\right]^{2}}{4 D_{0} t}\right) \\
& \times\left[\frac{x}{t}+D_{0}\left(\frac{F}{k T}+a\right)\right] .
\end{aligned}
$$

The general relation between the probability current and the particle current is given $[3,6]$ :

$$
\langle v\rangle_{t}=\int_{L} J(x, t) \mathrm{d} x .
$$

Note that $D$ is a very small value $\left(D_{0}=k T / \eta=\right.$ $\frac{k T}{6 \pi \mu R}[5,16]$, Boltzmann constant $K=1.3 \times 10^{-23} \mathrm{~J} / \mathrm{K}$, $T=300 \mathrm{~K}$, viscosity of water $\mu \approx 10^{-3} \mathrm{~Pa} \mathrm{~s}$, if radius of the particle is $R=200 \mathrm{~nm}, D \approx 10^{-12} \mathrm{~m}^{2} / \mathrm{s} \approx 1 \mu \mathrm{m}^{2} / \mathrm{s}$, in addition, in Ref. [10], $D_{0} \approx 2.7 \mu \mathrm{m}^{2} / \mathrm{s}$ ), for the convenience of physical discussion, we simulate the results with $D_{0}=1 \mu \mathrm{m}^{2} / \mathrm{s}$ and introduce a scaled force $f=F / k T$. In the adiabatic limit (temperature is constant) the scaled force $f$ only depends on the force of particle. The treatment does not affect the result analysis.

\section{Numerical simulation and discussion}

The probability density as a function of the $x$ for the different scaled force $f$ for a fixed channel geometry $\left(a=1 \mu \mathrm{m}^{-1}\right)$, is presented in Fig. 2. When $f=-1$, this corresponds to a diffusion in the absence of external force and entropy barrier. The peak of probability density is in the position of $x=0$. When $f=1$, the peak of probability density moves to the right in the position of $x=2$, it indicates that the probability of particle moving to the right is larger than to the left. When $f=0$, the situation of only pure entropy, peak of probability density moves to the right in the position of $x=1$. When $f=-2$, the peak of probability density moves to the left in the position of $x=-1$, it indicates that the probability of particle moving to the left is larger than to the right. According to Eq. (5) and Fig. 2, we can find that the peak of probability density will move with a velocity $D_{0}(f+a)$ in the $x$ direction.

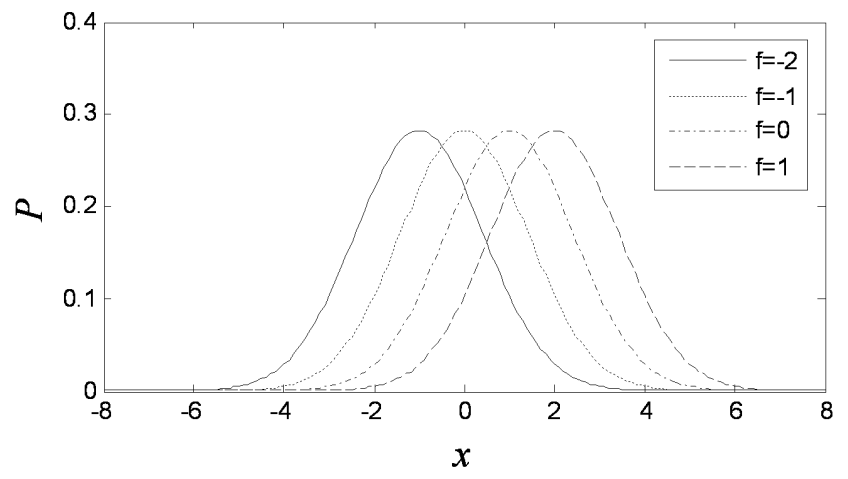

Fig. 2. Probability density $P$ vs. $x(\mu \mathrm{m})$ for different $f$ at $a=1 \mu \mathrm{m}^{-1}, t=1 \mathrm{~s}$.

The ratio $p$ as a function of $x$ for the different force parameter $f$ for a fixed channel geometry $\left(a=1 \mu \mathrm{m}^{-1}\right)$, is presented in Fig. 3a. Because this ratio does not depend on time and only involves the free energy, it can preferably show the particle motility. When $f=-a$, $P(x, t) / P(-x, t)=1$, it shows that probability of particle moving to the right is the same with the left, this corresponds to a unbiased diffusion in the absence of an external force and entropy barrier. When $f>-a$, 

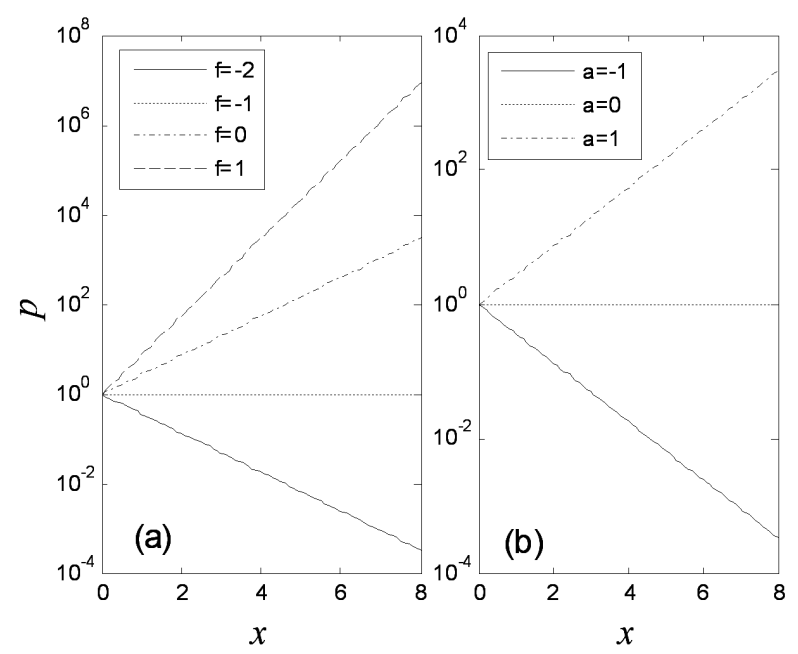

Fig. 3. (a) The ratio $p$ vs. $x(\mu \mathrm{m})$ for different $f$ at $a=1 \mu \mathrm{m}^{-1}$; (b) The ratio $p$ vs. $x(\mu \mathrm{m})$ for different $a\left(\mu \mathrm{m}^{-1}\right)$ at $f=0$.

$P(x, t) / P(-x, t)>1$ it shows that probability of particle moving to the right is larger than to the left, particle will move to the right. When $f<-a, P(x, t) / P(-x, t)<1$, it shows that probability of particle moving to the left is larger than to the right, particle will move to the left. Figure $3 \mathrm{~b}$ plots the ratio $p$ vs. the $x$, in the absence of an external force $(f=0)$, for the different $a$. When $a>0$, $P(x, t) / P(-x, t)>1$, it shows that probability of particle moving to the right is larger than to the left, particle will move to the right. When $a<0, P(x, t) / P(-x, t)<1$, it shows that probability of particle moving to the left is larger than to the right, particle will move to the left. When $a=0, P(x, t) / P(-x, t)=1$, this is a unbiased diffusion in the straight channel. This relation indicates that the entropy barrier will bias the diffusion of particle, from which we can know that the complex channel can give rise to multiple entropy barrier, which plays an important role in the particle motility. As the further description of the particle motility, the probability current density is illustrated schematically with Figs. 4-6.

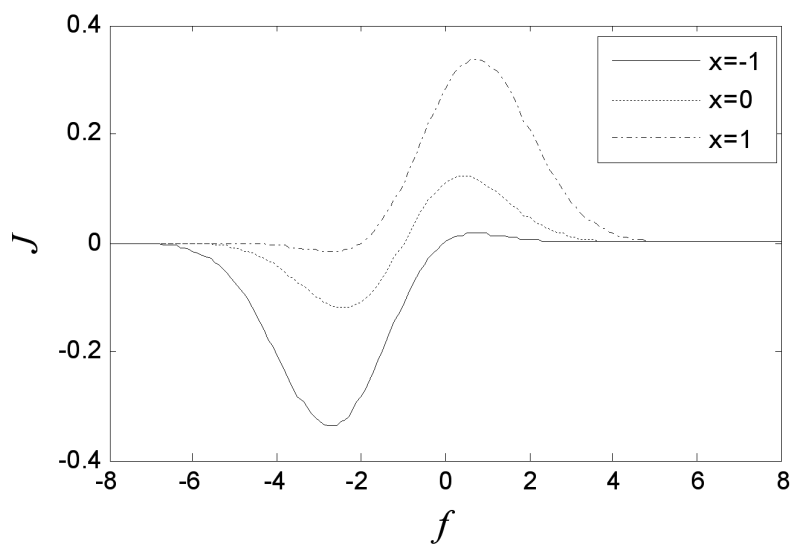

Fig. 4. Probability current density $J$ vs. $f$ for different $x(\mu \mathrm{m})$ at $a=1 \mu \mathrm{m}^{-1}, t=1 \mathrm{~s}$.
Figure 4 depicts the probability current density vs. the $f$, for a fixed channel geometry $\left(a=1 \mu \mathrm{m}^{-1}\right)$ and at $t=1$, for the different $x$. At a fixed position, $J$ will reverse its direction with the change of $f$. The probability current density takes its positive maximum value and the negative maximum for the corresponding $f$. Figure 5 plots the probability current density vs. the $t$, in the same geometry $\left(a=1 \mu \mathrm{m}^{-1}\right)$ and at $x=0$, for the different $f$. When $f=-a, J=0$, it indicates that probability density distribution will not shift with time increasing. When $f>-a, J>0$, it indicates that probability density distribution will move to the right with the time increasing. When $f<-a, J<0$, it indicates that probability density distribution will move to the left with the time increasing. Figure 5 shows that $J$ will tend to zero with the time increasing, it indicates that the probability density distribution will tend to be stable.

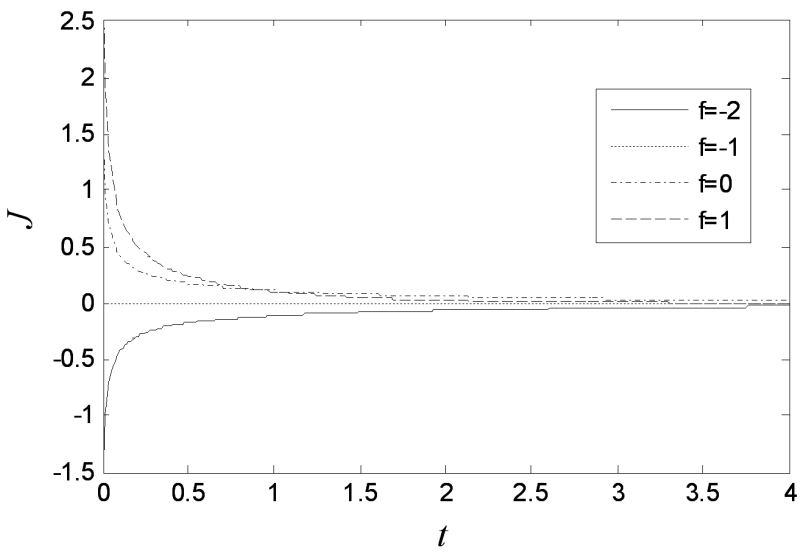

Fig. 5. Probability current density $J$ vs. $t$ (s) for different $f$ at $a=1 \mu \mathrm{m}^{-1}, x=0 \mu \mathrm{m}$.

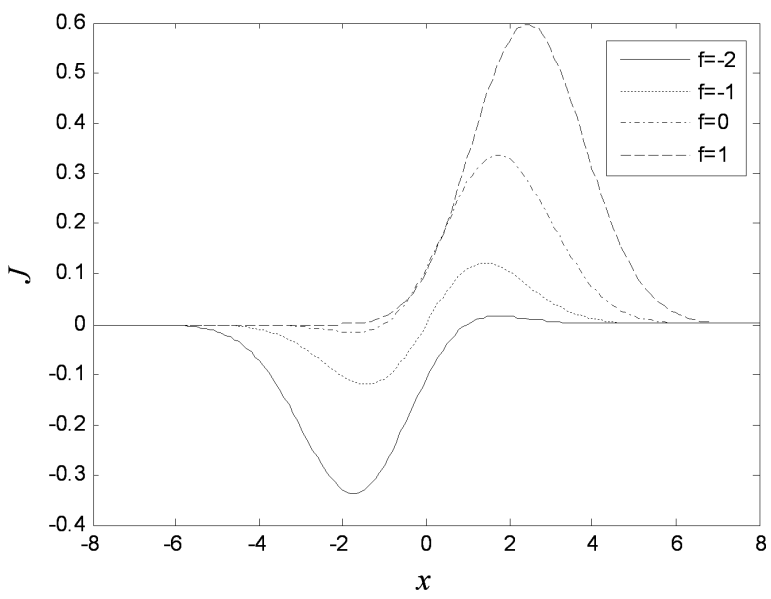

Fig. 6. Probability current density $J$ vs. $x(\mu \mathrm{m})$ for different $f$ at $a=1 \mu \mathrm{m}^{-1}, t=1 \mathrm{~s}$.

Figure 6 plots the probability current density vs. $x$, in the same geometry $\left(a=1 \mu \mathrm{m}^{-1}\right)$ and at $t=1$, for the different $f$. When the $f$ equals to $-1,0$ and $1, J$ will reverse with the change of $x$. The probability current density takes its positive maximum value and the negative 


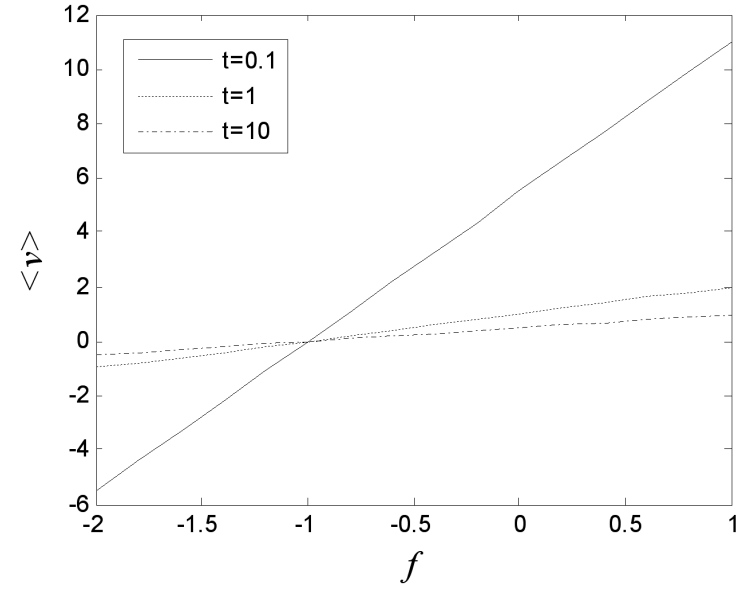

Fig. 7. Particle current $\langle v\rangle$ vs. $f$ for different time $t$ (s) at $a=1 \mu \mathrm{m}^{-1}$.

maximum for the corresponding $x$. According to Eq. (9) and Fig. 6, we can find: $f=-1$, particle current $\langle v\rangle=0$; $f=0$ and $f=1$, particle current is positive; $f=-2$, particle current is negative. The average particle current is illustrated schematically with Fig. 7.

Figure 7 depicts the particle current vs. the $f$, for a fixed geometry $\left(a=1 \mu \mathrm{m}^{-1}\right)$, for the different time $t$. Note that the direction of particle current does not change with increasing time $t$, just the size changes. When $f=-a$, particle current $\langle v\rangle=0$. When $f>-a$ particle current is positive, the particle will move to the right. When $f<-a$, particle current is negative, the particle will move to the left. The magnitude of particle current depends linearly on the strength of the $f$. In this way, one can separate particles by different forces and make them move in opposite directions. For instance, the splitting effect can be used for separation of particles (with same size) with different charge. For a device with $L=10 \mathrm{~mm}$ and the same geometry and parameters (for example $a=1 \mu \mathrm{m}^{-1}$ ) as in Fig. 1, with an external static electric field $E$ of $50 \mathrm{~V} / \mathrm{cm}$ in positive direction of $x$, the scaled force $f_{i}=F_{i} / k T$, here $F_{i}=q_{i} \times E$ is the force of particle of different charge. Particles of large negative charge $\left(q_{i}<-5 e, e=1.6 \times 10^{-19} \mathrm{C}\right)$ will move to the left with a velocity because of $f_{i}<-a\left(a=1 \mu \mathrm{m}^{-1}\right)$, whereas particles of small negative charge $\left(q_{i}>-5 e\right)$ or positive charge will drift to the right with a velocity because of $f_{i}>-a\left(a=1 \mu \mathrm{m}^{-1}\right)$. In addition, the entropy barrier plays a dominant role in particle splitting effect external force and entropy barrier can be used to separate particles of different size (with different diffusion coefficient and driven force) in a periodic channel [10, 17]. We can control the motion of Brownian particle by the splitting effect.

\section{Conclusion}

In summary, the transport of Brownian particle in a $3 \mathrm{D}$ throat-like channel with an external driving force is studied. In the fixed geometry channel, the external force can modify the motility of Brownian particle. A variation of the channel cross-sectional area can modify the entropy barrier, increasing (or decreasing) the entropy of particle, which can affect the mobility of particle. The transport of Brownian particle can be tuned by the entropy barrier or the external force. The results can be applied to particle separation techniques, Brownian motors, detection and analysis of biomolecules.

\section{Acknowledgments}

This work is supported by the Guizhou Science and Technology Foundation of China (Grant No. QKH J[2013]2241, QKH J[2013]2253), the International Science and Technology Cooperation Program of China (Grant No. 2014DFA00670).

\section{References}

[1] D. Reguera, G. Schmid, P.S. Burada, J.M. Rubi, P. Reimann, P. Hanggi, Phys. Rev. Lett. 96, 130603 (2006).

[2] H.Z. Xie, B.Q. Ai, X.M. Liu, X.B. Cheng, L.G. Liu, Z.B. Li, Chin. Phys. Lett. 24, 3340 (2007).

[3] P.S. Burada, G. Schmid, D. Reguera, J.M. Rubi, P. Hanggi, Phys. Rev. E 75, 051111 (2007).

[4] S. Martens, A.V. Straube, G. Schmid, L. SchimanskyGeier, P. Hanggi, Phys. Rev. Lett. 108, 010601 (2013).

[5] W. Riefler, G. Schmid, P.S. Burada, P. Hanggi, J. Phys. Condens. Matter 22, 454109 (2010).

[6] S. Martens, G. Schmid, L. Schimansky-Geier, P. Hanggi, Phys. Rev. E 83, 051135 (2011).

[7] A.M. Berezhkovskii, M.A. Pustovoit, S.M. Bezrukov, J. Chem. Phys. 126, 134706 (2007).

[8] V.Y. Zitserman, A.M. Berezhkovskii, A.E. Antipov, Y.A. Makhnovskii, J. Chem. Phys. 135, 121102 (2011).

[9] N. Laachi, M. Kenward, E. Yariv, K.D. Dorfman, Europhys. Lett. 80, 50009 (2007).

[10] D. Reguera, A. Luque, P.S. Burada, G. Schmid, J.M. Rubi, P. Reimann, P. Hanggi, Phys. Rev. Lett. 108, 020604 (2012).

[11] E. Yariv, K.D. Dorfman, Phys. Fluids 19, 037101 (2007).

[12] A.G. Marin, J.M. Sancho, Phys. Rev. E 77, 031108 (2008).

[13] R. Zwanzig, J. Phys. Chem. 96, 3926 (1992).

[14] D. Reguera, J.M. Rubi, Phys. Rev. E 64, 061106 (2001).

[15] M.V. Vazquez, A.M. Berezhkovskii, L. Dagdug, J. Chem. Phys. 129, 046101 (2008).

[16] P. Reimann, C. Van den Broeck, H. Linke, P. Hanggi, J.M. Rubi, A. Perez-Madrid, Phys. Rev. E 65, 031104 (2002).

[17] K.J. Huang, S.J. Qin, Z.C. Bai, X. Zhang, J.D. Mai, J. Appl. Phys. 114, 194702 (2013). 